\title{
ANALYSIS OF THE DYNAMIC PERFORMANCE OF SERIAL 3R ORTHOGONAL MANIPULATORS
}

\author{
Dinh Quan Nguyen \\ Sébastien Briot \\ Institut de Recherches en Communications et Cybernétique
de Nantes (IRCCyN), UMR CNRS 6597
1 rue de la Noë, BP 92101, 44321 Nantes Cedex 3 France
}

Philippe Wenger

\section{ABSTRACT}

Serial 3R orthogonal manipulators have been studied recently and it has been proved that they can exhibit good performances in term of workspace size and kinematic properties. The aim of this work is to analyze their dynamic performances, and compare them with anthropomorphic manipulators, which are very popular in industry.

Static and dynamic analyses based on the evaluation of the maximal input torques required for moving the manipulator are achieved. It is shown that, as in kinematics, the dynamic performances of the serial $3 \mathrm{R}$ orthogonal manipulators are better.

\section{INTRODUCTION}

Nowadays most industrial manipulators are based on anthropomorphic architectures (i.e. PUMA type). These manipulators are composed of a vertical revolute joint linked to the ground followed by two parallel joints and a spherical wrist. But other joint arrangements exist such as for the $3 R$ orthogonal manipulators: any two consecutive joint axes are orthogonal. There are few examples of this type of manipulators such as the IRB $6400 \mathrm{C}$ by ABB-Robotics launched in 1998 (not longer commercialized) and the DIESTRO manipulator built at McGill University. But it appears that, due to the success of anthropomorphic manipulators, 3R orthogonal manipulators have almost disappeared.

Recently, researchers showed more and more interests in this kind of manipulators. It has been shown that these manipulators may have quite different kinematic properties according to their link lengths and joint offsets. The late studies, [1]-[3], exposed deeply the analysis of geometric and kinematic properties of this manipulator family. For example the determination of the workspace boundary, the size and shape of the workspace, the existence of holes and voids, the accessibility inside the workspace, the feasibility of continuous trajectories in the workspace, singularity curves, and other properties like "cuspidality" (possibility of changing posture without crossing a singularity) were well-discussed.

In [4], the orthogonal manipulators and the PUMA-type manipulators were compared in terms of kinematic performances. The aim of this paper is to continue the comparison between these two manipulators based on the analysis on their dynamic performances.

Until now, the fields related to dynamic performances of a manipulator are still opened. The motion of a manipulator, a multi-body mechanical system, involves several effects caused by inertia, centrifugal, Coriolis, gravity and friction forces/ torques. Most of these are nonlinear effects, which makes very difficult to predict and control the dynamic performances of the manipulator. Several measures of dynamic performances of manipulators have been introduced in the literature. The simplest indices to define and understand are probably the maximal input torques or joint acceleration that are required when the manipulator is moving, or also the dissipated energy. But as they depend on the manipulator trajectory, general conclusions may be difficult to give.

In order to avoid these drawbacks, Asada [5] introduced the generalized inertia ellipsoid (GIE) as a tool to measure the capability of changing the end-effectors velocity in different directions for fixed kinetic energy. Yoshikawa [6] proposed to use the dynamic manipulability ellipsoid (DME) for measuring the ease of changing the end-effector configuration by a set of joint torques with fixed magnitude (Euclidean norm). Both the GIE and the DME are based on the relationship between the generalized inertia force of the endeffector and the generalized inertia torques of joints. Tourassis and Neuman [7] investigated the inertial characteristics of the dynamics equations of manipulators. They introduced the coefficients of coupling to measure the structural coupling of the dynamics equations. Khatib and Burdick [8] derived a dynamic model in operational space, which is, in fact, the

\footnotetext{
* Author of correspondence, Phone: +33 2403769 47, Fax: +33 2403769 30, Email: Philippe.Wenger@irccyn.ec-nantes.fr
} 
space spanned by the vector representing the pose of the endeffector. Using the operational space approach, they presented an optimization method for finding the largest and uniform bounds of end-effector's acceleration corresponding to the minimum joint torques at both low and high bounds of joint velocities. Graettinger and Krogh [9] introduced another performance measure termed acceleration radius. For given bounds of joint torques, the corresponding acceleration radius defines the minimum upper bound of the magnitude of endeffector acceleration over the whole workspace. Other measures that attempt to capture manipulator performances as a function of the dynamics can be cited: Bowling and Khatib [10] proposed a general framework for capturing the dynamic capability of a general manipulator that includes the velocity and acceleration characteristics of the end-effector, taking into account factors such as torque and velocity limits of the actuators. Herman [11] presented several indices for the evaluation of nonlinear effects during the motion of a manipulator based on the kinetic energy, based on the equations of motion expressed in term of inertial quasivelocities. Rao and Bhatti [12] proposed a method to construct manipulator kinematic and dynamic model using a probabilistic approach to study the effect of various factors causing uncertainties in the behavior of a manipulator.

All these indices have the same advantage: they do not depend on the manipulator trajectory. However, they may be difficult to understand by engineers. The goal of this paper is to propose a general optimization procedure based on the analysis of the manipulator maximal input torques during motion, for characterizing the dynamic performances of the manipulators under study.

The paper is organized as follows. In the second part, the manipulators under study are presented. The third section shows results from static analysis. Then our optimization procedure is defined and applied to verify the dynamic performances. Last section concludes this paper.

\section{DESCRIPTION OF THE MANIPULATORS UNDER STUDY}

This section introduces the geometric properties and kinematic performances of the PUMA and 3R Orthogonal (ORTH) manipulators.

\subsection{Serial 3R orthogonal manipulators}

\subsubsection{Geometric description}

The orthogonal manipulators are positioning manipulators with three revolute joints in which the two pairs of adjacent joint axes are orthogonal. Figure 1 shows examples of the architecture of the manipulators and their modified DenavitHartenberg parameters [13] are presented in table 1.

Recent studies [2]-[4] have shown that, by simply setting certain geometric parameters to zero, it is possible to achieve different sub-families for the orthogonal manipulator with different kinematic performances, especially in their workspace topology (Fig. 2).

It is possible to show that the PUMA manipulator exhibits similar properties. Therefore among these many sub-families of each manipulator, it is necessary to choose the most suitable ones in order to perform the comparison between them.
Table 1. Geometric parameters of ORTH manipulator

\begin{tabular}{|c|c|c|c|c|c|}
\hline Joint & $\boldsymbol{\sigma}$ & $\boldsymbol{\alpha}$ & $\mathbf{d}$ & $\boldsymbol{\theta}$ & $\mathbf{r}$ \\
\hline 1 & 0 & 0 & 0 & $\theta_{1}$ & 0 \\
\hline 2 & 0 & $-90^{0}$ & $\mathrm{~d}_{2}$ & $\theta_{2}$ & $\mathrm{r}_{2}$ \\
\hline 3 & 0 & $90^{0}$ & $\mathrm{~d}_{3}$ & $\theta_{3}$ & $\mathrm{r}_{3}$ \\
\hline
\end{tabular}



Figure 1. Geometric structure of 3R orthogonal manipulators

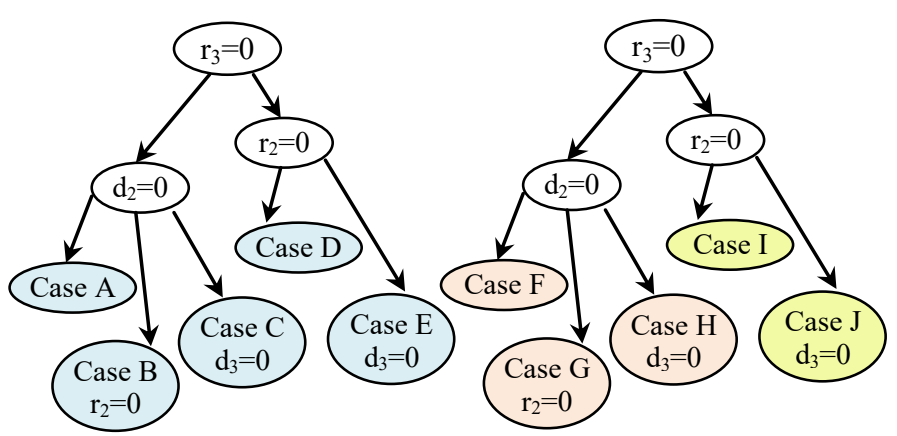

Figure 2. The ten families of manipulators with at least one parameter equal to zero [2]

\subsubsection{Kinematic performances analysis}

The kinematic performances of 3R ORTH manipulator have been studied in [4] based on the analysis of the regular dexterous workspace (RDW).

RDW is a part of the working area - free of singularities and with a regular shape (cube, square...) - within which, the dexterity is better than a minimal prescribed value $\lambda$. The dexterity can be assessed with the reciprocal of the conditioning index $k^{-1}$ of the Jacobian matrix $\mathbf{J}$ :

$$
k=\sigma_{\max } / \sigma_{\min }
$$

where, $\sigma_{\max }$ and $\sigma_{\min }$ are the maximum and minimum singular values of $\mathbf{J}$.

In order to calculate the RDW, for example for a square shape, one can first find the maximal square inscribed in the workspace cross section (Fig. 3a):

$$
a=2 \min (d)
$$

where $d$ is the Chebyshev distance from the center point $\mathrm{M}_{0}$ to a singular point $\mathrm{A}_{\mathrm{s}}$ :

$$
d=\max \left(\left|\rho_{0}-\rho_{s}\right|,\left|z_{0}-z_{s}\right|\right)
$$

then find the maximal sub-square where $k^{-1}>\lambda$ where $\lambda$ is a prescribed value that is up to the designer (Fig. 3b).

In [14] a performance index was defined that accounts for workspace compactness and dexterity:

$$
\eta=a_{R D W} / \rho_{\max }
$$





Figure 3. RDW with $\mathrm{k}_{\min }^{-1}=0.25$ (ORTH type $C: \mathrm{d}_{4}=1.5, \mathrm{r}_{2}=1$ ) [4]

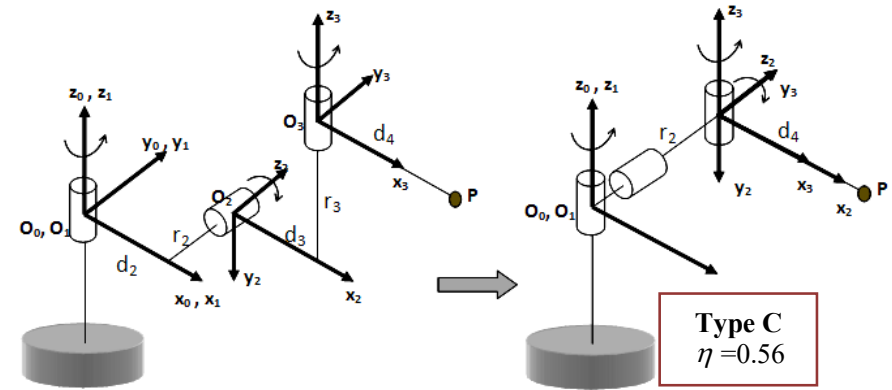

Figure 4. Optimized ORTH manipulator, $\left(r_{3}=0, r_{2}=d_{4}, d_{2}=0\right)$

where, $a_{R D W}$ is the side's length of the regular dexterous workspace section and $\rho_{\max }$ is the maximal reach of the manipulator $\left(\rho=\sqrt{x^{2}+y^{2}}\right.$, with $(x, y, z)$ are the coordinates of the end-effector in the task-space).

It is shown in [4] that the ORTH manipulator with the maximum value of performance index $\eta$ has the following proper geometric parameters:

$$
d_{2}=0, d_{3}=0, r_{3}=0, r_{2}=d_{4}
$$

Its forward kinematic equations are given by:

$\left\{\begin{array}{l}x=d_{4} C 3 C 2 C 1-d_{4}(1+S 3) S 1 \\ y=d_{4} C 3 C 2 S 1-d_{4}(1+S 3) C 1 \\ z=-d_{4} C 3 S 2\end{array}\right.$

where $C i=\cos \left(\theta_{i}\right), S i=\sin \left(\theta_{i}\right)$

Its inverse kinematic equations are given by

$$
\left\{\begin{array}{l}
\theta_{3}=\operatorname{asin}\left(\frac{x^{2}+y^{2}+z^{2}}{2 d_{4}^{2}}-1\right) \rightarrow 2 \text { solutions } \\
\theta_{2}=\operatorname{asin}\left(\frac{-z}{d_{4} C 3}\right) \rightarrow 2 \text { solutions } \\
\theta_{1}=\operatorname{atan} 2\left(\frac{C 3 C 2 y-(1+S 3) x}{C 3 C 2 x+(1+S 3) y}\right)
\end{array}\right.
$$

In this case, because of the simplicity in geometric structure, four distinct Inverse Kinematic Solutions (IKS) of ORTH manipulator result into two groups with equivalent postures (Fig. 5).

The selected optimized ORTH manipulator belongs to type C with the performance index $\eta=0.56$ :

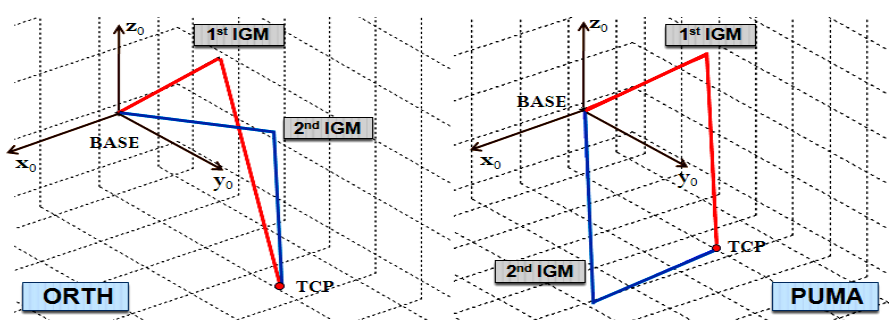

Figure 5. Postures of ORTH_VER and PUMA at an example point in the workspace

\subsection{Description of the PUMA manipulator}

Figure 6 shows examples of the architecture of the manipulators and their modified Denavit-Hartenberg parameters [13] are presented in table 2 .

Applying the same procedure for optimizing the kinematic performances of the PUMA manipulator leads to quite interesting results. The PUMA manipulator has very close performance index value and the same geometric parameters (link lengths) as the optimized ORTH one [4]:

$$
d_{2}=0, r_{2}=0, r_{3}=0, d_{3}=d_{4}
$$

Its forward kinematic equations are given by

$$
\begin{aligned}
& x=\left[d_{3} C 2+d_{4} C 23\right] C 1-\left(r_{2}+r_{4}\right) S 1 \\
& \left\{y=\left[d_{3} C 2+d_{4} C 23\right] S 1+\left(r_{2}+r_{4}\right) C 1\right. \\
& z=-d_{3} S 2-d_{4} S 23 \\
& S 23=\sin \left(q_{2}+q_{3}\right), \ldots \\
& \rightarrow\left\{\begin{array}{l}
x=d_{4}[C 2+C 23] C 1 \\
y=d_{4}[C 2+C 23] S 1 \\
z=-d_{4}(S 2+S 23)
\end{array}\right.
\end{aligned}
$$

and its inverse kinematic equations are

$$
\begin{cases}\theta_{1}=\operatorname{atan} 2\left(\frac{y}{x}\right) & \rightarrow 2 \text { solutions } \\ \theta_{3}=\operatorname{asin}\left(\frac{x^{2}+y^{2}+z^{2}}{2 d_{4}^{2}}-1\right) & \rightarrow 2 \text { solutions } \\ \theta_{2}=\operatorname{atan} 2\left(\frac{-z C 1(1+C 3)-x S 3}{-z C 1 S 3+x(1+C 3)}\right) & \end{cases}
$$

In this case, the PUMA manipulator also has 4 IKS solutions and results into two groups of equivalent postures.

For easiest notation later, we will indicate the two groups of equivalent postures of each manipulator as follows: $\mathbf{1}^{\text {st }}+\mathbf{2}^{\text {nd }}$ IGM ORTH and $\mathbf{1}^{\text {st }}+2^{\text {nd }}$ IGM PUMA (here, $1^{\text {st }}$ group of PUMA manipulator has the elbow-up posture, $2^{\text {nd }}$ group has the elbow-down posture - Fig. 5).

The workspace of ORTH manipulator is a torus (simply rotate a sphere with radius $d_{4}$ around $\mathbf{z}$-axis of the first joint) while PUMA's workspace is a sphere with radius $2 \times d_{4}$ (Fig. 7).

The selected optimized PUMA manipulator has the performance index $\eta=0.6$.

2.3 Manipulator choice

The mass and link-length parameters for the selected ORTH manipulator and PUMA manipulator are chosen such that they are similar to the parameters of first three links of the real 
Table 2. Geometric parameters of PUMA manipulator

\begin{tabular}{|c|c|c|c|c|c|}
\hline Joint & $\boldsymbol{\sigma}$ & $\boldsymbol{\alpha}$ & $\mathbf{d}$ & $\boldsymbol{\theta}$ & $\mathbf{r}$ \\
\hline 1 & 0 & 0 & 0 & $\theta_{1}$ & 0 \\
\hline 2 & 0 & $-90^{0}$ & 0 & $\theta_{2}$ & $\mathrm{r}_{2}$ \\
\hline 3 & 0 & 0 & $\mathrm{~d}_{3}$ & $\theta_{3}$ & 0 \\
\hline
\end{tabular}


Figure 6. Optimized PUMA manipulator, $(d 2=d 3=r 3=r 2=0$; $d 3=d 4)$



Figure 7. Workspace of ORTH and PUMA manipulators

PUMA-560 manipulator [15] in order to make the fairest comparison as possible, i.e. the link lengths are $r_{2}=d_{4}=0.433$ $\mathrm{m}$, and the dynamic parameters are shown in tables 3 and 4 . In these tables, $\mathrm{Mj}$ represents the mass of link $j^{\text {th }}(j=1,2,3)$, $(\mathrm{MXj}, \mathrm{MYj}, \mathrm{MZj})$ are their first moments of inertia and $(\mathrm{XXj}, \mathrm{XYj}, \mathrm{XZj}, \mathrm{YYj}, \mathrm{YZj}, \mathrm{ZZj})$ are second moments of inertia [13].

\subsection{Dynamic modelling of ORTH and PUMA manipulators}

The inverse dynamic model (IDM) of a rigid manipulator composed of $n$ moving links calculates the motor forces/torques vector, as a function of the generalized coordinates and their derivatives. It can be obtained from the Newton-Euler or the Lagrangian equations [13]:

$$
\boldsymbol{\Gamma}_{d y n}=\boldsymbol{\Gamma}_{i n}+\boldsymbol{\Gamma}_{f}
$$

where:

$\boldsymbol{\Gamma}_{d y n}$ is the $(n \times 1)$ vector of dynamic forces/torques due to the inertial, centrifugal, Coriolis and gravitational effects:

$$
\boldsymbol{\Gamma}_{d y n}=\mathbf{M}(\mathbf{q}) \ddot{\mathbf{q}}+\mathbf{C}(\mathbf{q}, \dot{\mathbf{q}}) \dot{\mathbf{q}}+\mathbf{Q}(\mathbf{q})
$$

$\mathbf{q}, \dot{\mathbf{q}}, \ddot{\mathbf{q}}$ are respectively the $(n \times 1)$ vectors of generalized joint positions, velocities and accelerations,

$\mathbf{M}(\mathbf{q})$ is the $(n \times n)$ manipulator inertia matrix,

$\mathbf{C}(\mathbf{q}, \dot{\mathbf{q}})$ is the $(n \times n)$ matrix of centrifugal and Coriolis effects,

$\mathbf{Q}(\mathbf{q})$ is the $(n \times 1)$ vector of gravitational forces/torques,
Table 3. Dynamic parameters of ORTH manipulator: payload $=\mathbf{5 k g}$

\begin{tabular}{|c|c|c|c|c|c|c|c|c|c|c|}
\cline { 2 - 10 } & $\begin{array}{c}\mathbf{X X j} \\
\left(\mathrm{kgm}^{2}\right)\end{array}$ & $\begin{array}{c}\mathbf{X Y j} \\
\left(\mathrm{kgm}^{2}\right)\end{array}$ & $\begin{array}{c}\mathbf{X Z j} \\
\left(\mathrm{kgm}^{2}\right)\end{array}$ & $\begin{array}{c}\mathbf{Y Y j} \\
\left(\mathrm{kgm}^{2}\right)\end{array}$ & $\begin{array}{c}\mathbf{Y Z j} \\
\left(\mathrm{kgm}^{2}\right)\end{array}$ & $\begin{array}{c}\mathbf{Z Z j} \\
\left(\mathrm{kgm}^{2}\right)\end{array}$ & $\begin{array}{c}\mathbf{M X j} \\
(\mathrm{kgm})\end{array}$ & $\begin{array}{c}\mathbf{M Y j} \\
(\mathrm{kgm})\end{array}$ & $\begin{array}{c}\mathbf{M Z j} \\
(\mathrm{kgm})\end{array}$ & $\begin{array}{c}\mathbf{M j} \\
(\mathrm{kg})\end{array}$ \\
\hline $\mathbf{1}$ & 0.0166 & 0 & 0 & 0.0166 & 0 & 0.0166 & 0 & 0 & 0 & 4.43 \\
\hline $\mathbf{2}$ & 0.6438 & 0 & 0 & 0.6438 & 0 & 0.0128 & 0 & 0 & -2.208 & 10.2 \\
\hline $\mathbf{3}$ & 0.0120 & 0 & 0 & 1.1330 & 0 & 1.1324 & 2.8051 & 0 & 0 & 9.80 \\
\hline
\end{tabular}

Table 4. Dynamic parameters of PUMA manipulator: payload = $5 \mathrm{~kg}$

\begin{tabular}{|c|c|c|c|c|c|c|c|c|c|c|}
\cline { 2 - 10 } \multicolumn{1}{c|}{} & $\begin{array}{c}\mathbf{X X j} \mathbf{j}^{2} \\
\left(\mathrm{kgm}^{2}\right)\end{array}$ & $\begin{array}{c}\mathbf{X Y j} \\
\left(\mathrm{kgm}^{2}\right)\end{array}$ & $\begin{array}{c}\mathbf{X Z j} \\
\left(\mathrm{kgm}^{2}\right)\end{array}$ & $\begin{array}{c}\mathbf{Y Y j} \\
\left(\mathrm{kgm}^{2}\right)\end{array}$ & $\begin{array}{c}\mathbf{Y Z j} \\
\left(\mathrm{kgm}^{2}\right)\end{array}$ & $\begin{array}{c}\mathbf{Z Z j} \\
\left(\mathrm{kgm}^{2}\right)\end{array}$ & $\begin{array}{c}\mathbf{M X j} \\
(\mathrm{kgm})\end{array}$ & $\begin{array}{c}\mathbf{M Y j} \\
(\mathrm{kgm})\end{array}$ & $\begin{array}{c}\mathbf{M Z j} \\
(\mathrm{kgm})\end{array}$ & $\begin{array}{c}\mathbf{M j} \\
(\mathrm{kg})\end{array}$ \\
\hline $\mathbf{1}$ & 0.0166 & 0 & 0 & 0.0166 & 0 & 0.0166 & 0 & 0 & 0 & 4.43 \\
\hline $\mathbf{2}$ & 0.0128 & 0 & 0 & 0.4845 & 0 & 0.4845 & 2.2083 & 0 & 0 & 10.2 \\
\hline $\mathbf{3}$ & 0.0120 & 0 & 0 & 1.1330 & 0 & 1.1324 & 2.8051 & 0 & 0 & 9.80 \\
\hline
\end{tabular}

$\boldsymbol{\Gamma}_{i n}$ is the $(n \times 1)$ input electromagnetic force/torque vector of the drive chain, in SI units on the joint side,

$\Gamma_{f}$ is the $(n \times 1)$ vector of the loss force/torque due to frictions and motor iron losses, eddy currents and hysteresis effect. Usually, it is approximated with a viscous friction coefficient and a Coulomb friction force/torque:

$$
\boldsymbol{\Gamma}_{f}=-\mathbf{F}_{v} \dot{\mathbf{q}}-\mathbf{F}_{c} \operatorname{sign}(\dot{\mathbf{q}})-\mathbf{F}_{\text {coff }}
$$

where:

$\mathbf{F}_{v}$ is the $(n \times n)$ diagonal matrix of viscous parameters,

$\mathbf{F}_{c}$ is the $(n \times n)$ diagonal matrix of dry friction parameters, and $\operatorname{sign}($.$) denotes the sign function,$

$\mathbf{F}_{\text {coff }}$ is the $(n \times 1)$ vector of asymmetrical Coulomb friction force/torque between positive and negative velocities.

Thus (11) becomes:

$$
\boldsymbol{\Gamma}_{d y n}=\boldsymbol{\Gamma}_{i d m}-\mathbf{F}_{v} \dot{\mathbf{q}}-\mathbf{F}_{c} \operatorname{sign}(\dot{\mathbf{q}})-\boldsymbol{\Gamma}_{o f f}
$$

where:

$\boldsymbol{\Gamma}_{\text {off }}$ is the $(n \times 1)$ vector of offset force/torque that regroups the amplifier offset and the asymmetric Coulomb friction coefficient,

$\boldsymbol{\Gamma}_{i d m}$ defines the motor force/torque which includes the amplifier offset component,

Then the inverse dynamic model (IDM) is given by:

$$
\boldsymbol{\Gamma}_{i d m}=\mathbf{M}(\mathbf{q}) \ddot{\mathbf{q}}+\mathbf{N}(\mathbf{q}, \dot{\mathbf{q}})
$$

$\mathbf{N}(\mathbf{q}, \dot{\mathbf{q}})$ regroups the $(n \times 1)$ vector of centrifugal, Coriolis, gravitational, friction and offset forces/torques:

$$
\mathbf{N}(\mathbf{q}, \dot{\mathbf{q}})=\mathbf{C}(\mathbf{q}, \dot{\mathbf{q}}) \dot{\mathbf{q}}+\mathbf{Q}(\mathbf{q})+\mathbf{F}_{v} \dot{\mathbf{q}}+\mathbf{F}_{c} \operatorname{sign}(\dot{\mathbf{q}})+\boldsymbol{\Gamma}_{\text {off }}
$$

Computing these dynamic models is generally tedious, therefore a software, namely SYMORO+ [16] has been used. In the remainder, the static and dynamic models of ORTH and PUMA manipulators are computed thanks to SYMORO+.

\section{STATIC ANALYSIS}

In this section, the static analysis of the two manipulators is presented. This analysis gives a first insight of the 
manipulator performance but is also used for the optimization procedure presented in the next part.

The static analysis is a special case where the actuator torques are computed from the IDM with $\dot{\mathbf{q}}=\mathbf{0}$ and $\ddot{\mathbf{q}}=\mathbf{0}$.

As a result, the input torques value only depends on the manipulator configuration. Therefore, for having a general insight of the manipulator performances in static, the input torques are good indicators.

Because of symmetry, it is only necessary to consider a cross-section of the workspace (a circle with radius $R=d_{4}$ ). Figure 8 shows an example of the static torques profile (absolute values) of the two manipulators (with payload $=$ $5 \mathrm{~kg}$ ) for the first IKS solution.

Figures 9 and 10 show the isocontour plots in the workspace of the maximum and minimum static torque between the two IKS of each manipulator. Then the changes of maximum torque absolute values with respect to the changes of the payload (for all IKS) are shown in figure 11. In these figures, T1,T2,T3 are the actuator torques of joint 1,2 and 3 of each manipulator respectively.

The static analysis gives much information:

- For the ORTH manipulator, static torque profiles for maximum and minimum of absolute values of the input torques are similar (this was expected since the two postures of the ORTH manipulator are close while those of the PUMA manipulator are quite different)

- The joint torques 2 and 3 of ORTH manipulator are distributed quite equally. Meanwhile for the PUMA, the torque values of joint 2 are much larger (more than twice than joint 3)

To sum up, in static analysis, the ORTH manipulator exhibits better performance than the PUMA manipulator.

\section{DYNAMIC ANALYSIS}

As presented in the introduction, the main drawback of analyzing the dynamic performances with the manipulator input torques is that they depend on the trajectory. In order to avoid this problem, one idea could be to make the manipulator moves on selecting "exciting" trajectories that make it exhibiting the highest values of the input torques. To generate these exciting trajectories, it is necessary to define an optimization procedure that is detailed below.

In this section, the following assumptions are made:

- The analysis is performed under the same trajectories in the task-space for both manipulators, with the same payloads and under the same maximum operational velocity $\mathrm{V}=$ const (magnitude of Tool Control Point's (TCP) velocity in the task-space).

- The traveling time between any two points A and B in the task-space is known.

- The manipulator keeps track of the trajectory perfectly, i.e. the controller is considered as ideal. Hence, all the terms are computed with desired joint positions, velocities and accelerations $\left(\mathbf{q}^{d}, \dot{\mathbf{q}}^{d}, \ddot{\mathbf{q}}^{d}\right)$.

- In the dynamic models, we do not consider the effects of frictions (FV, FS).

- Because ORTH manipulator has two equivalent postures which are close to each other (Fig. 5) and the PUMA

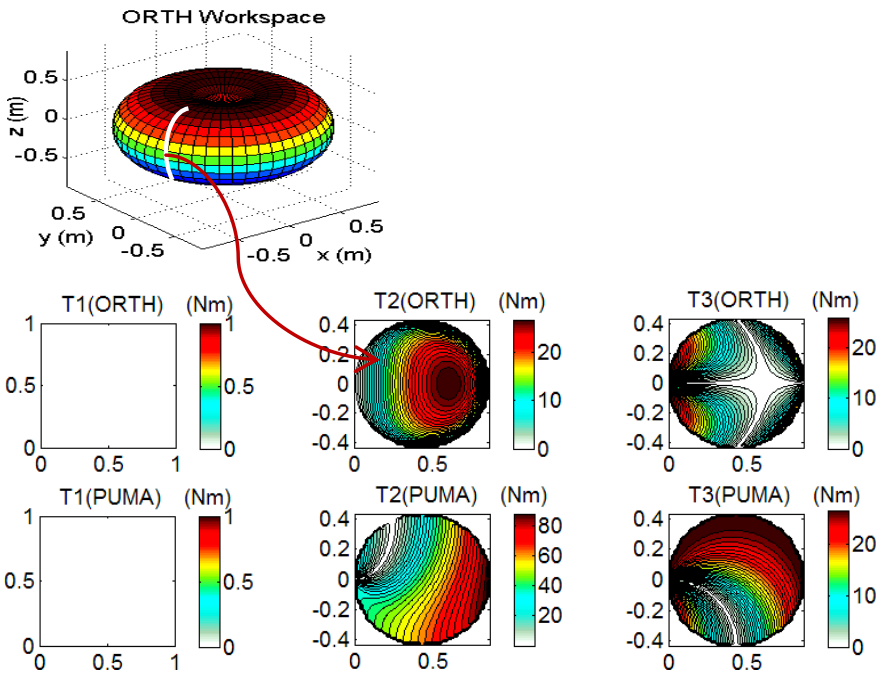

Figure 8. Profiles of the static torques absolute values for ORTH and PUMA manipulators for the first IKS solution (payload $=\mathbf{5 k g}$ )
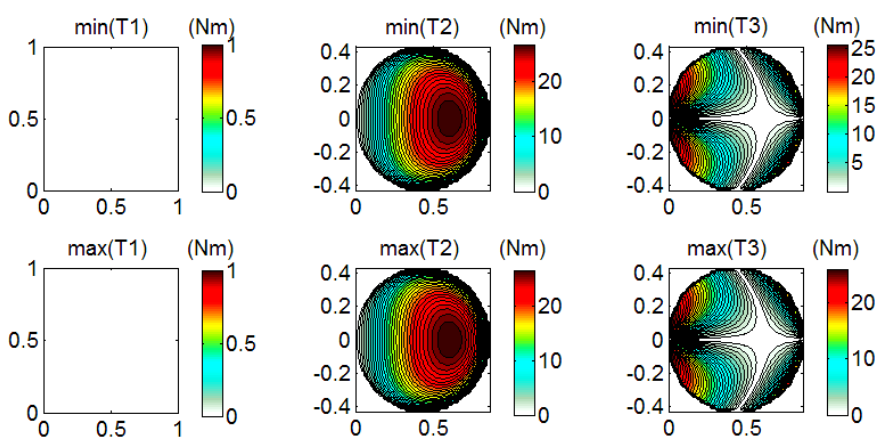

Figure 9. Profiles of the static torques absolute values for ORTH manipulator (payload $=5 \mathrm{~kg}$ )
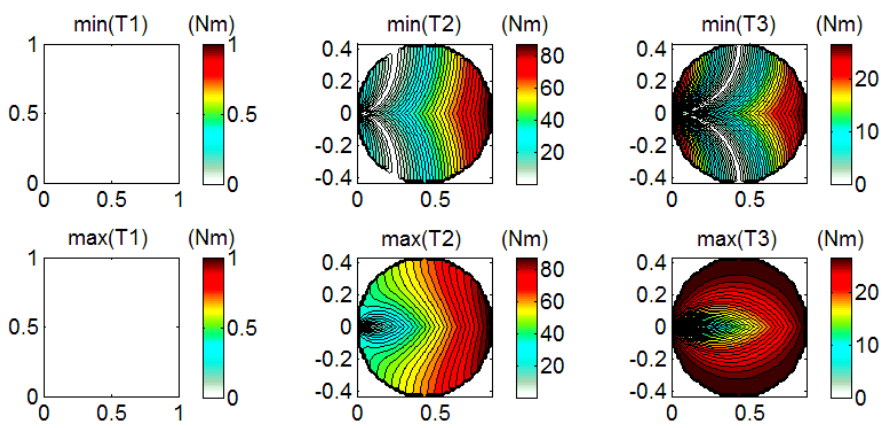

Figure 10. Profiles of the static torques absolute values for PUMA manipulator (payload $=5 \mathbf{k g}$ )
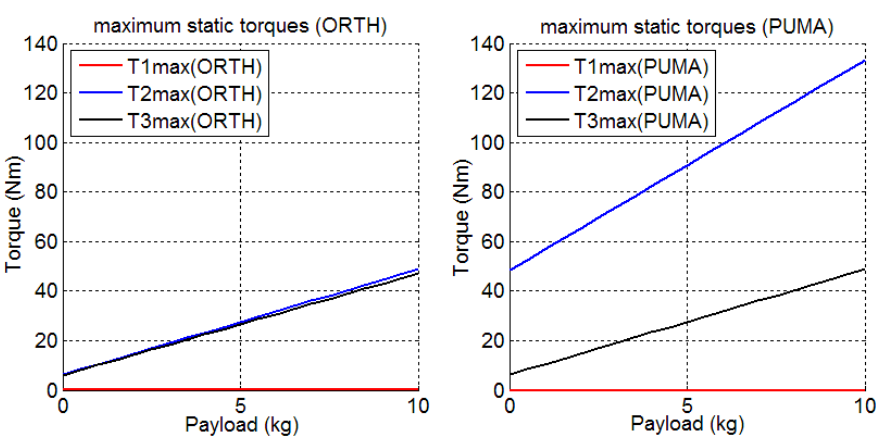

Figure 11. Comparison of maximum absolute values of the static torques between PUMA and ORTH manipulators 


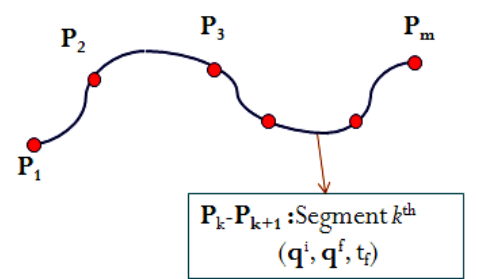

Figure 12. Example of optimized trajectory

manipulator also has one similar elbow-up posture $\left(1^{\text {st }}\right.$ IGM) hence we will only consider the comparison results between $\left(1^{\text {st }}\right.$ IGM of ORTH manipulator $)$ and $\left(1^{\text {st }}\right.$ IGM of PUMA manipulator).

\subsection{Optimization procedure}

\section{Trajectory generation}

Several methods for exciting trajectory generation exist (most of them have been developed for dynamic parameter identification) [17]. It is proposed here to adapt one of them in order to generate the desired exciting trajectories that enable the manipulators to attain their maximal input torques.

A desired trajectory is composed of several segments which are determined by a subset of optimized points. Each via-point on the trajectory are constrained to belong to different areas in the workspace. These areas are chosen thanks to the static analysis that defined some zones where the static torques are high. It is necessary to choose different zones that allow covering all the workspace. Hence a suitable method to put the desired points is needed. Two methods can be used to link these points :

- Point-to-Point: the trajectory will be constructed from several segments, i.e. $\mathbf{P}_{1}-\mathbf{P}_{2}, \mathbf{P}_{2}-\mathbf{P}_{3}, \mathbf{P}_{3}-\mathbf{P}_{4}, \mathbf{P}_{4}-\mathbf{P}_{5}$, etc. The manipulator starts at the beginning of each segment and stops at its extremity. Different motion profiles can be applied on these paths $\left(5^{\text {th }}\right.$ order polynomial laws, bangbang profiles, etc.)

- Via-Points: the trajectory is constructed using a continuous spline defined by a subset of several points, i.e. $\mathbf{P}_{1}-\mathbf{P}_{2}-\mathbf{P}_{3}-\mathbf{P}_{4}-\mathbf{P}_{5}-\ldots-\mathbf{P}_{\text {n }}$. The manipulator starts at $\mathbf{P}_{1}$ and only stops at point $\mathbf{P}_{\mathrm{n}}$. In the remainder of the paper, cubic splines are used [13].

\section{Choosing an optimization tool}

All the analysis steps were carried out by using the software MATLAB which provides several optimization toolboxes for solving single-objective problems or multiobjectives problems (i.e. fmincon, fgoalattatin...).

In this case, we choose fgoalattain function to perform the optimization tasks since several objectives will be considered.

\section{Optimization algorithm}

With the purpose of comparing, the optimization procedure is performed in order to define the trajectories in the task-space of the ORTH or PUMA manipulator. At first, it is important to select which posture of each manipulator will be compared. After achieving the optimized profile (that consists of several points and travelling time) for one manipulator, the corresponding inverse-solution of the other manipulator can be found.
By doing so, the constraints of the end-effector's position inside the ORTH workspace can be set in order not to cross the singularity boundary (especially for PUMA manipulator). Checking whether or not the trajectory is inside the working space is also needed for desired via-points which are close to the boundary.

The procedure to construct exciting trajectories is detailed as follows:

\section{Objective:}

$$
\sum_{k} a b s\left(T_{j \mathrm{kmax}}\right) \rightarrow \max , \text { for } j=1,2,3
$$

where the subscript $k$ corresponds to the $k^{\text {th }}$ segment of the trajectory (Fig. 12).

$$
\begin{aligned}
& \text { Searching parameters: } \\
& \qquad \mathbf{X}=(\mathbf{P s}, \mathbf{t}) \rightarrow N_{X}=4 m-1
\end{aligned}
$$

Assume that the optimized trajectory has $m$ points whose coordinates are stored in the vector Ps. Then the decision variables $\mathbf{X}$ is a column vector with the length of $N_{X}$ (the first $(m-1)$ elements are travelling time and the following $3 m$ elements are coordinates of $m$ via-points in the task-space).

\section{Constraints:}

$$
\begin{aligned}
& \left\{\begin{array}{l}
\left|\mathrm{T}_{\mathrm{ikmax}}\right| \leq \mathrm{T}_{\text {imax }} \quad(\mathrm{i}=1,2,3) \\
\mathbf{V}(k)=\frac{\mathbf{L}(\mathrm{k})}{\mathrm{t}_{\Sigma}} \leq \mathbf{V}_{\max }(k) \quad(k=1,2, \ldots, m-1) \\
\mathbf{0} \leq \mathbf{t}(\text { travelling time must be positive }) \\
\mathbf{P}(\mathrm{x}, \mathrm{y}, \mathrm{z}) \in \text { ORTH's workspace }
\end{array}\right. \\
& \left\{\begin{array}{l}
\mathbf{x}_{k \min } \leq \mathbf{x}_{k} \leq \mathbf{x}_{k \max } \\
\mathbf{y}_{k \min } \leq \mathbf{y}_{k} \leq \mathbf{y}_{k \max }(\text { constraint limits in task-space) } \\
\mathbf{z}_{k \min } \leq \mathbf{z}_{k} \leq \mathbf{z}_{k \max }
\end{array}\right.
\end{aligned}
$$

where:

$N_{X}$ is the total number of searching parameters,

$\mathrm{T}_{\text {imax }}$ is the maximal torque value of joint $i^{\text {th }}$,

$\mathrm{T}_{\mathrm{ikmax}}$ is the maximal torque value of joint $i^{\text {th }}$ at segment $k^{\text {th }}$,

$\mathbf{V}_{\max }$ is the $(1 \times \mathrm{m}-1)$ vector of maximal velocities of the endeffector at all segments,

$\mathbf{L}(k)$ is the total travelling length of the end-effector at segment $k^{\text {th }}$ :

$$
\mathbf{L}(\mathrm{k})=\sum\|\mathbf{P}(\mathrm{n}+1)-\mathbf{P}(\mathrm{n})\|
$$

$\mathrm{t}_{\Sigma}=\mathbf{t}(\mathrm{k})$ is the total traveling time at segment $k^{\text {th }}$,

$\mathbf{P}(x, y, z)$ is position of the end-effector in the task-space.

\section{Determine the limit ranges}

Choosing initial starting points is one important factor of the optimization procedure. Here, the static torques profiles (see section 3) are used to determine ( $\mathbf{X}_{k \text { min }}, \mathbf{X}_{k \text { max }}$ ).

The idea is to select the limit ranges that could cover the entire workspace and also that could pass through the most exciting zones defined by static analysis (i.e. from low distributing torques areas to high distributing torques areas). One way to do so is to divide the workspace into small elements (i.e. small cubes).

The workspace can be divided into layers (separated by the segments 1 to 32 on Fig. 13) regrouped in 4 regions (I,II,III,IV). Each layer is a cross-section of the ORTH's 
workspace and is indicated with an index $i(i=1,2, \ldots, 32-$ indices on the largest external circle of the torus). A crosssection is divided into 32 squares. Each square is indicated with an index $j(j=1,2, \ldots, 32)$. A center point $\mathbf{P}(i, j)$ of a searching zone is defined as the center of the corresponding square with index $j$ on the layer with index $i$. Coordinates of this point in the task-space can be obtained by:

$$
\mathbf{P}_{i, j}=\operatorname{Rot}\left(z, \theta_{i}\right) \times \mathbf{P}_{0, j}
$$

where $\theta_{i}=i \times(\pi / 16), \mathbf{P}_{0, j}(x, 0, z)$ is the center point of the $j^{t h}$ square on the layer with index $0 / 32$, and $\operatorname{Rot}\left(z, \theta_{i}\right)$ denotes the rotation matrix along the z-axis by an angle $\theta_{i}$.

A trajectory with desired passing locations and directions can then be constructed by choosing suitable indices $\left(i_{k}, j_{k}\right)$ for any via-point $\mathbf{P}_{k}$.

In optimization, the starting points are initialized as random values within offsets around the center points $\mathbf{P}_{k}\left(x_{k}, y_{k}, z_{k}\right)$. Then the procedure will search for optimized points inside the limit cubes defined by (18).

\subsection{Optimization results}

Four different optimized trajectories are generated using 5 via-points - i.e. 4 segments - (total 19 searching parameters) depending on the four regions of the workspace (I,II,III,IV). In table 5:

- The matrix $\mathbf{P}$ contains the initial parameters used for the localization of the center via-points. Initial parameters of point $\mathbf{P}_{k}$ are given by the $k^{\text {th }}$ column of $\mathbf{P}$. The first row contains indices for layers $-i_{k}$, the second row contains indices for squares $-j_{k}$.

- The $k^{\text {th }}$ term of vector $\Delta$ represents the size $\Delta_{k}$ of the searching interval for the final position of point $\mathbf{P}_{k}$ (Fig.13).

- The maximum torques (T1, T2, T3) are chosen based on the static torque profiles with a payload of $5 \mathrm{~kg}$.

Figure 13 shows an example of constructing the range's limit for any trajectory with desired locations and directions. For region $\mathrm{I}$, we have:

$$
\mathbf{P}:\left[\begin{array}{ccccc}
1 & 7 & 3 & 2 & 6 \\
22 & 16 & 5 & 27 & 13
\end{array}\right], \quad \boldsymbol{\Delta}:\left[\begin{array}{ccccc}
\frac{\mathrm{d}_{4}}{6} & \frac{\mathrm{d}_{4}}{6} & \frac{\mathrm{d}_{4}}{6} & \frac{\mathrm{d}_{4}}{6} & \frac{\mathrm{d}_{4}}{3}
\end{array}\right]
$$

This means that the center desired via-points are:

$$
\mathbf{P}_{\mathbf{1}}(1,22)-\mathbf{P}_{\mathbf{2}}(7,16)-\mathbf{P}_{\mathbf{3}}(3,5)-\mathbf{P}_{\mathbf{4}}(2,27)-\mathbf{P}_{\mathbf{5}}(6,13)
$$

Test 1: optimization using Cubic Spline function (viapoints): optimization for ORTH manipulator first

In this test, the optimization procedure for the ORTH manipulator is first performed to bring out its performance in the worst possible cases (reach constraint boundaries of maximum torques or maximum speed). Then, the same trajectory of the TCP is applied to the PUMA manipulator.

Figures $14-16$ show the results of the optimized trajectories with joint accelerations and joint torques of each manipulator by using cubic spline function method (via-points method).

Test 2: optimization using $5^{\text {th }}$ degree polynomial (point-topoint): optimization for PUMA manipulator first

In this test, the optimization procedure for the PUMA manipulator is first performed to bring out its performance in the worst possible cases. Then, the same trajectory of the TCP is applied to the ORTH manipulator.

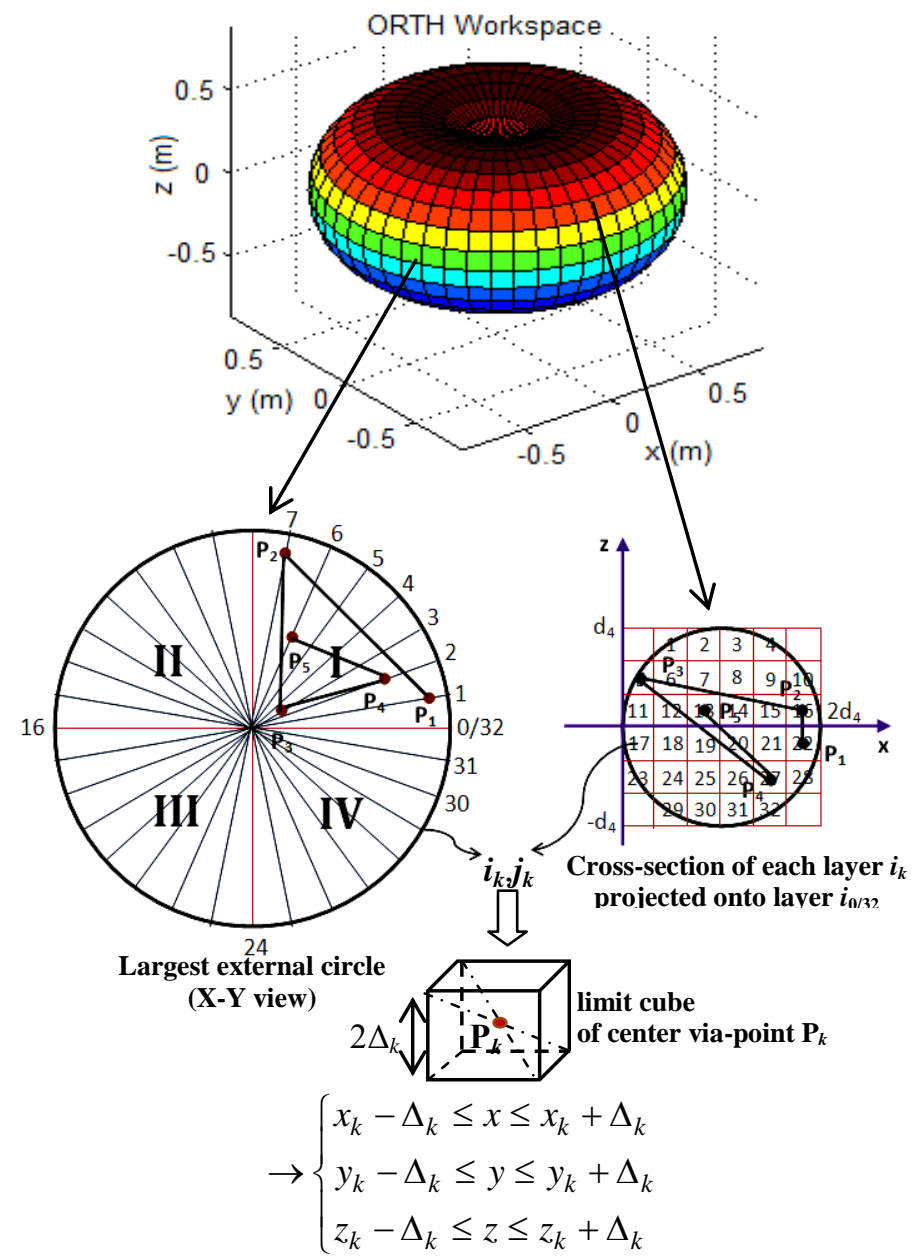

Figure 13. Constructing limit ranges for desired trajectory



Figure 14. Optimized Trajectories : Cubic Spline, task-space

(Test 1 - optimization for ORTH first)

Figures 17 - 19 show the results of the optimized trajectories with joint accelerations and joint torques of each manipulator by using $5^{\text {th }}$ degree polynomial method (point-topoints method).

In term of joint torques, the ORTH manipulator has better performance. The joint torques values of the PUMA in both cases are larger, especially much larger in case of joint 2 (Fig. $16,19)$. In term of joint accelerations, both manipulators have fair performances (Fig. 15, 18). 


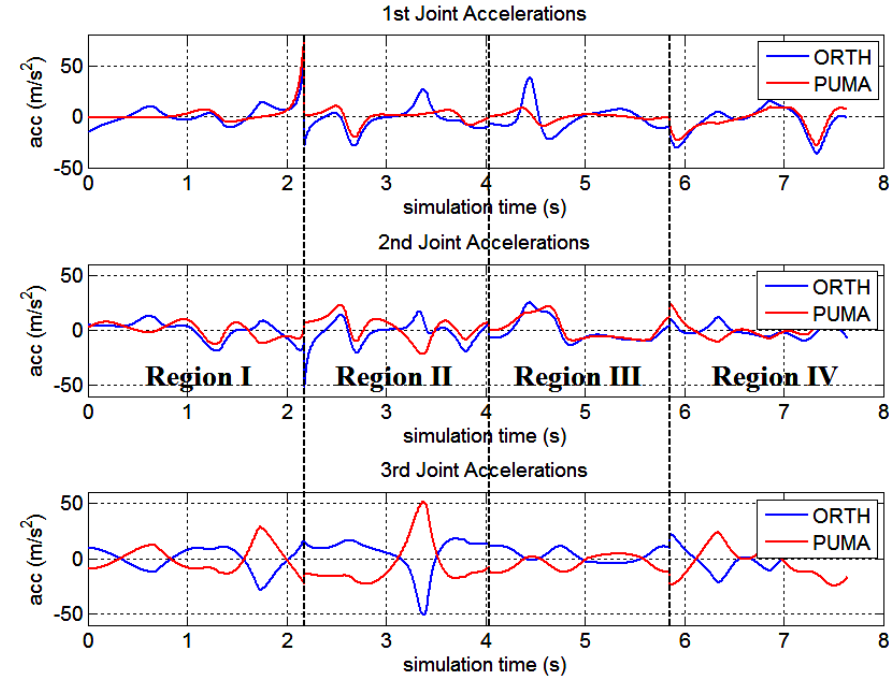

Figure 15. Joint Accelerations : Cubic Spline, task-space (Test 1 - optimization for ORTH first)

Table 5. Initial parameters for optimization procedure

\begin{tabular}{|c|c|c|}
\hline & \multicolumn{2}{|c|}{ Center Via-Points } \\
\hline & Cubic spline function & $5^{\text {th }}$ degree polynomial \\
\hline I & 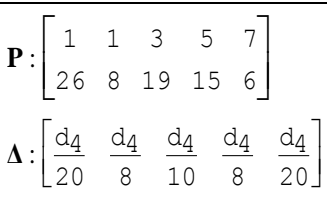 & $\begin{array}{l}\mathbf{P}:\left[\begin{array}{ccccc}1 & 7 & 3 & 2 & 6 \\
22 & 16 & 5 & 27 & 13\end{array}\right] \\
\Delta:\left[\begin{array}{lllll}\frac{d_{4}}{6} & \frac{d_{4}}{6} & \frac{d_{4}}{6} & \frac{d_{4}}{6} & \frac{d_{4}}{3}\end{array}\right]\end{array}$ \\
\hline II & 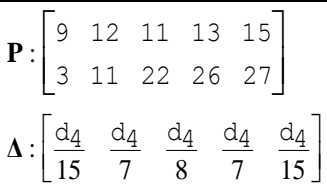 & 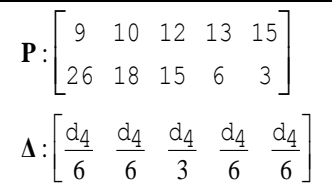 \\
\hline III & $\begin{array}{l}\mathbf{P}:\left[\begin{array}{ccccc}17 & 19 & 20 & 21 & 23 \\
9 & 6 & 24 & 25 & 13\end{array}\right] \\
\Delta:\left[\begin{array}{lllll}\frac{d_{4}}{6} & \frac{d_{4}}{7} & \frac{d_{4}}{6} & \frac{d_{4}}{7} & \frac{d_{4}}{6}\end{array}\right]\end{array}$ & 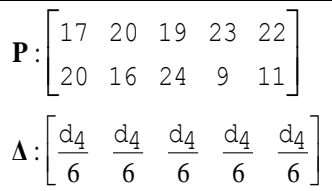 \\
\hline IV & 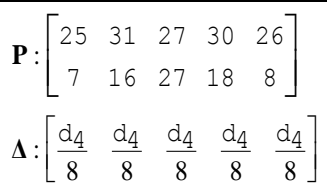 & $\begin{array}{l}\mathbf{P}:\left[\begin{array}{ccccc}25 & 27 & 26 & 29 & 31 \\
2 & 22 & 24 & 3 & 20\end{array}\right] \\
\Delta:\left[\begin{array}{lllll}\frac{d_{4}}{5} & \frac{d_{4}}{5} & \frac{d_{4}}{5} & \frac{d_{4}}{5} & \frac{d_{4}}{5}\end{array}\right]\end{array}$ \\
\hline & $\begin{array}{c}\text { Max Torques } \\
{[\text { T1 T2 T3] }(\mathrm{Nm})}\end{array}$ & $\begin{array}{c}\text { Max TCP Speed } \\
{\left[\begin{array}{l}V_{1} \\
V_{2}\end{array} V_{3} V_{4}\right](\mathrm{m} / \mathrm{s})}\end{array}$ \\
\hline $\mathbf{I}$ & {$\left[\begin{array}{lll}80 & 80 & 30\end{array}\right]$} & 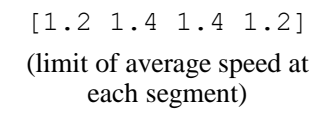 \\
\hline II & - & {$\left[\begin{array}{llll}1.3 & 1.3 & 1.3 & 1.3\end{array}\right]$} \\
\hline III & - & {$\left[\begin{array}{llll}1.2 & 1.2 & 1.2 & 1.2\end{array}\right]$} \\
\hline IV & - & {$\left[\begin{array}{llll}1.3 & 1.2 & 1.2 & 1.3\end{array}\right]$} \\
\hline
\end{tabular}

Indicating performance areas for optimized via-points

Figure $20-21$ show examples of maximum torques profile of the two manipulators for optimized via-points obtained from test 1 and test 2 . The maximal torque values of each joint for both manipulators are computed while changing

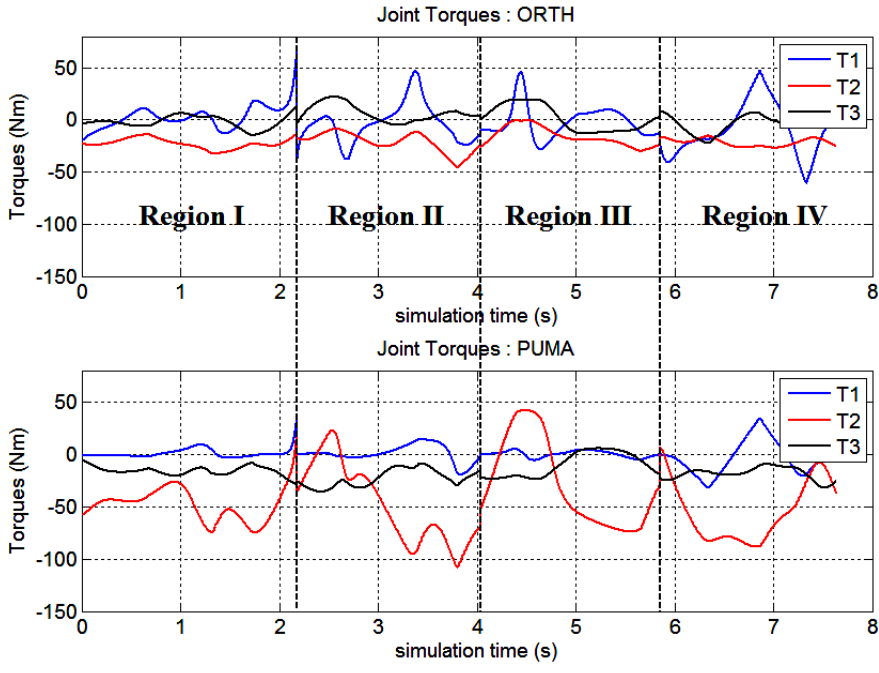

Figure 16. Actuator Torques : Cubic Spline, task-space (Test 1 - optimization for ORTH first)
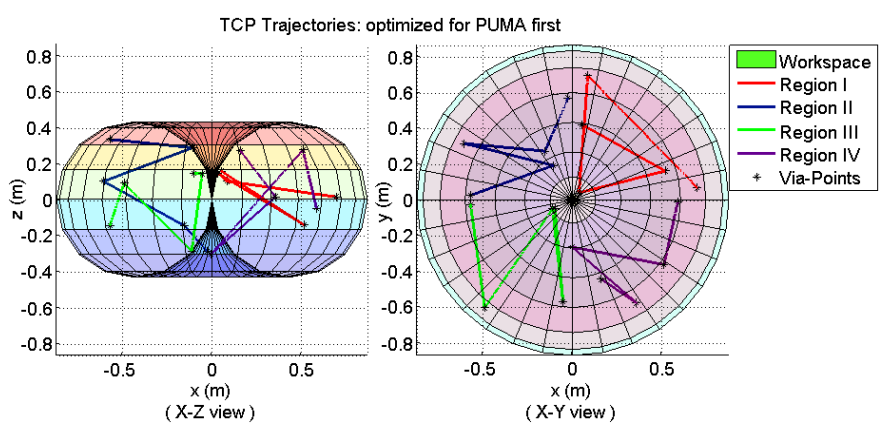

Figure 17. Optimized Trajectories : $5^{\text {th }}$ degree, task-space (Test 2 - optimization for PUMA first)

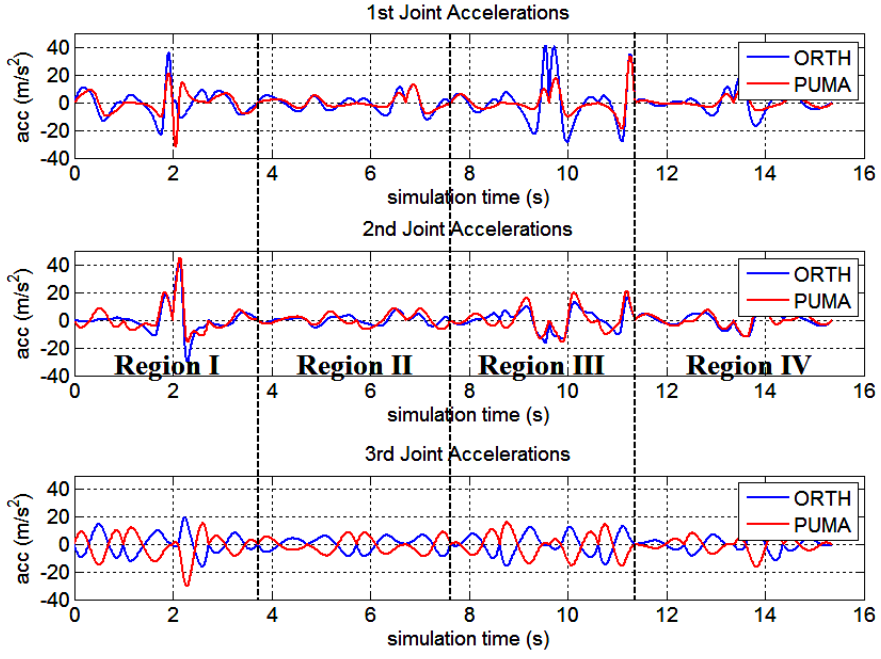

Figure 18. Joint Accelerations : $5^{\text {th }}$ degree, task-space (Test 2 - optimization for PUMA first)

the maximal values of the speed of the end-effector (Vs) and the payload.

Notice that each segment of a trajectory is composed of three terms: the initial point $\mathbf{P}^{\text {init }}$, the final point $\mathbf{P}^{\text {final }}$ and the travelling time $t_{f}$. One can change the speed of the TCP by varying the value of $t_{f}$ while keeping the via points fixed. 




Figure 19. Actuator Torques : $5^{\text {th }}$ degree, task-space (Test 2 - optimization for PUMA first)


Figure 20. Difference in maximum of the absolute values of the input torques between ORTH and PUMA manipulators (verifying with cubic spline function method for via-points in test 1 )

Because the torque profiles for each manipulator are complex surfaces (the maximal torque values are varied while changing either the speed Vs or payload), the contours plots of the differences between maximum torque values of the ORTH and the PUMA are presented. In the areas that have the negative values, the ORTH manipulator performs better than the PUMA manipulator.

In practice, it is enough to consider the areas with a velocity of the end-effector less than $1 \mathrm{~m} / \mathrm{s}$. The performances of joint torques 2 and 3 of the ORTH manipulator are better
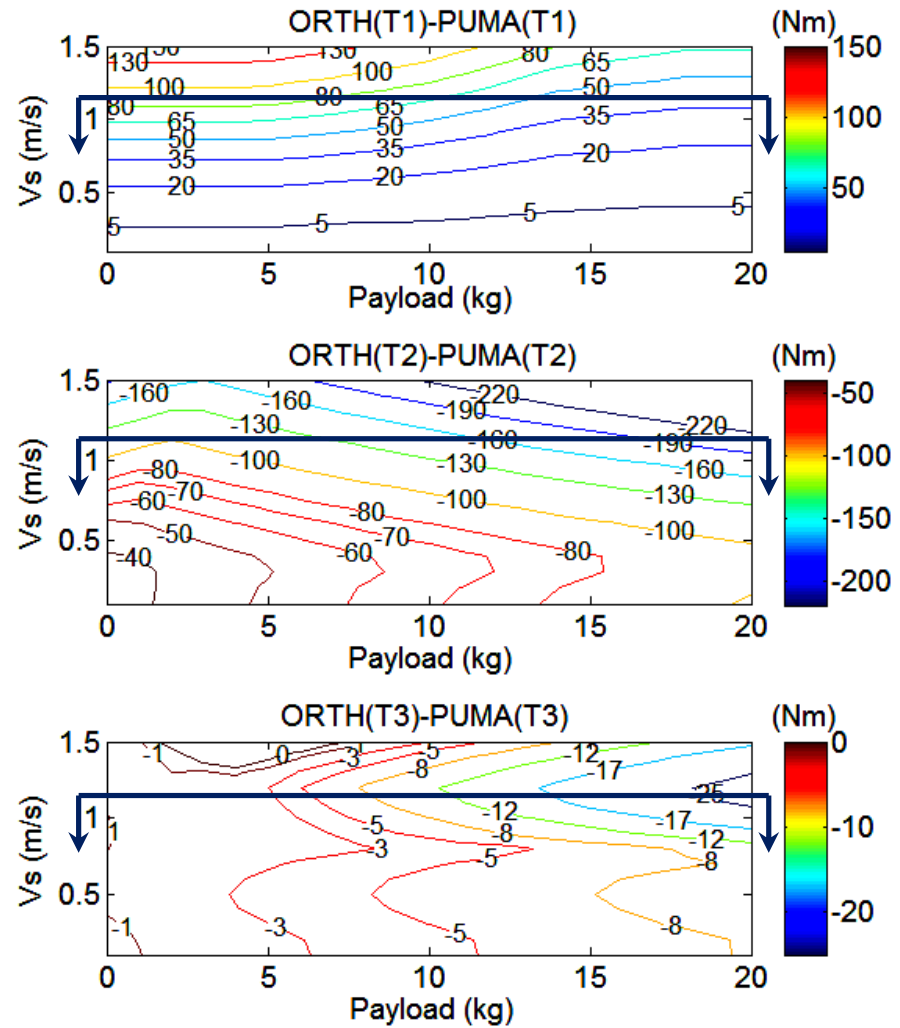

Figure 21. Difference in maximum of the absolute values of the input torques between ORTH and PUMA manipulators (verifying with $5^{\text {th }}$ degree polynomial method for via-points in test 2)

than for the PUMA manipulator. Although joint torque 1 of the ORTH manipulator is larger than for the PUMA, the differences are small with respect to the differences of the second joint torques.

\section{CONCLUSIONS}

The aim of this work was to analyze the dynamic performances of $3 \mathrm{R}$ orthogonal manipulators, and to compare them with anthropomorphic manipulators, which are very popular in industry.

Static and dynamic analyses based on the evaluation of the maximal input torques required for moving the manipulator have been achieved. Moreover, a systematic procedure for analyzing the dynamic performances of manipulators in term of input torques has been proposed. It has been shown that the dynamic performances of the serial 3R orthogonal manipulators are better than those of the PUMA manipulator.

\section{REFERENCES}

[1] P. Wenger: "Design of cuspidal and noncuspidal manipulators", Proceedings of IEEE International Conference on Robotics and Automation, 1997, pp. 2172 2177.

[2] M. Zein et al: "An exhaustive study of the workspace topologies of all 3R orthogonal manipulators with geometric simplifications", Sciendirect, Mechanism and Machine Theory 41, 2006, pp. 971-986 
[3] M. Baili: "Classification des manipulateurs 3R orthogonaux", PhD Thesis, Ecole Centrale de Nantes, 2004.

[4] M. Zein: “Analysis cinématique des manipulateurs sériels 3R orthogonaux et des manipulateurs parallèles plans", $\mathrm{PhD}$ Thesis, Ecole Centrale de Nantes, 2007.

[5] H. Asada: "A geometrical representation of manipulator dynamics and its application to arm design", Trans ASME, J. Dyn. Syst., Meas. and Control, Vol. 105, Sept. 1983, pp. 131-135.

[6] T. Yoshikawa: "Dynamic manipulability of robot manipulators", Proc. 24th ZEEE Conf. Decision and Control, 1985, pp. 1033-1038.

[7] V. D. Tourassis, C. P. Neuman: "The inertial characteristics of dynamic robot models", Mechanism and Machine Theory, Vol. 20, No. 1, 1985, pp.41-52.

[8] O. Khatib, J. Burdick: "Optimization of dynamics in manipulator design: the operational space formulation", Znt. J. Robotics and Auto., Vol.2, May 1987, No.2, pp. 90-98.

[9] T. J. Graettinger, B. H. Krogh: "The acceleration radius: a global performance measure for robotic manipulators", ZEEE J. Robotics and Auto., Vo1.4, No. 1, Feb. 1988, pp. 60-69.
[10]A. Bowling, O. Khatib: "The dynamic capability equations: a new tool for analyzing robotic manipulator performance", IEEE Trans. Robot. 21(1), 2005, pp. 115123.

[11]P. Herman: "Energy-Based Indices for Manipulator Dynamics Improvement", Springer, Journal of Intelligent and Robotic Systems, Vol. 44, 2005, pp. 313-325.

[12] S.S. Rao, P.K. Bhatti: "Probabilistic approach to manipulator kinematics and dynamics", Sciencedirect, Reliability Engineering and System Safety 72, 2001, pp. 47-58.

[13] W. Khalil, É. Dombre: "Modeling, Identification and Control of Robots", Kogan Page Science, 2004.

[14]F. Majou: "Analysis cinétostatique des machines parallèles à translations", $\mathrm{PhD}$ Thesis, Université Laval, Québec, 2004.

[15]P. Corke, P. I. Corke Brian Armstrong-h'elouvry: "A search for consensus among model parameters reported for the PUMA 560 robot", Proc. IEEE Int. Conf. Robotics and Automation, 1994, pp. 1608-1613.

[16] W. Khalil, D. Creusot: "SYMORO+: A System for the Symbolic Modelling of Robots", Robotica, Vol. 15, 1997, pp. 153-161.

[17] C. Pressé: "Identification des Paramétres Dynamiques des Robots", PhD Thesis, Ecole Centrale de Nantes, 1994. 